Revista Científica

https://revistacientifica.edu.pe/index.php/revistacientifica/article/view/9

Agosto 2021

Volumen 1 / No. 1

ISSN:

pp. $1-13$

https://revistacientifica.edu.pe

\title{
Actividad hidrolitica de la amilasa sanguinea en pacientes Altoandinos aparentemente sanos - Ayacucho
}

Hydrolytic activity of blood amylase in apparently healthy highAndean patients - Ayacucho

Atividade hidrolítica da amilase sangüínea em pacientes aparentemente saudáveis dos Altos Andes - Ayacucho

\section{ARTÍCULO GENERAL}

Dr. Jesús Javier Ñaccha Urbano

https://orcid.org/0000-0002-5035-4849

jesus.naccha@unsch.edu.pe

Laboratorio de Biología Humana. Facultad Ciencias Biológicas. UNSCH

Recibido 08 de Marzo 2021 | Arbitrado y aceptado 28 de Marzo 2021 | Publicado en 05 Abril 2021

\section{RESUMEN}

En el presente trabajo de investigación determinamos los niveles de amilasa sanguínea así como la variación de actividad amilásica sanguínea. De los 70 pacientes altoandinos, 35 mujeres cuyos promedios son: actividad amilásica fue de $125 \mathrm{UA} / \mathrm{dl}$, 26 años de edad y $58 \mathrm{Kg}$ de peso. En cuanto a grado de amilasa sanguínea en pacientes altoandinos aparentemente sanos de ambos sexos relación al índice de masa corporal, se muestra que en su mayoría de los jóvenes presentan un normopeso: 18 pacientes de mujeres $120.6 \mathrm{UA} / \mathrm{dl}$ pero estadísticamente no significativa.

Palabras claves: Amilasa, sangre, pancreatitis, UA/dl.

\section{ABSTRACT}

In the present research work we determined the levels of blood amylase as well as the variation of blood amylase activity. Of the 70 high Andean patients, 35 women whose averages are: amylase activity was $125 \mathrm{AU} /$ dl, 26 years of age and $58 \mathrm{~kg}$ of weight. Regarding the degree of blood amylase in apparently healthy high Andean patients of both sexes in relation to the body mass index, it is shown that most of the young people present a normal weight: 18 female patients 120.6 AU / dl but not statistically significant.

Key words: Amylase, blood, pancreatitis, UA / dl.

\section{RESUMO}

No presente trabalho de pesquisa, determinamos os níveis de amilase no sangue, bem como a variação da atividade da amilase no sangue. Dos 70 pacientes andinos altos, 35 mulheres cujas médias são: a atividade da amilase foi $125 \mathrm{UA} / \mathrm{dl}$, 26 anos de idade e $58 \mathrm{~kg}$ de peso. Em relação ao grau de amilase sanguínea em pacientes andinos altos aparentemente saudáveis de ambos os sexos em relação ao índice de massa corporal, mostra-se que a maioria dos jovens apresenta peso normal: 18 pacientes do sexo feminino $120,6 \mathrm{UA} /$ dl mas não estatisticamente significante.

Palavras-chave: Amilase, sangue, pancreatite, UA / dl. 


\section{INTRODUCCIÓN}

Jesús Javier Ñaccha Urbano

La base de la dieta alimentaria del poblador andino (actualmente si tiene las condiciones socio económicas adecuadas) está constituida por los carbohidratos en un $70 \%$ las proteínas en $20 \%$ por lípidos en un $10 \%$ y las vitaminas en $3 \%$ y minerales en un $2 \%$. La mayoría de los productos contiene gran contenido de polímeros de almidones que se encuentran en cereales (maíz, trigo y cebada) y tubérculos (papa, camote, oca, olluco, aracacha, yuca, mashua), leche de vacuno y caprino, carne de animales (res, cordero, chancho, caprino, cuy, alpaca y pescado) pocas frutas (pacae, chirimoya, guinda, aguaymanto, tuna y cítricos) y minerales (cucurbitáceas, coca y plantas medicinales).

Sabemos bioquímicamente que mediante las vías metabólicas se transforman en glucógeno en el interior de los músculos útil para el trabajo arduo del agricultor como del pequeño ganadero o pastor. El papel biológico del almidón es almacenarse en diversas estructuras vegetales que posteriormente al consumir consume es catabolizado por una enzima especifica conocido como amilasa (contiene alfa y beta amilasa), generándose una reacción hidrolítica para obtener la maltosa un disacárido conocido.

La amilasa enzima primordial lo podemos encontrar en la saliva, sangre, orina, inclusive en la cavidad peritoneal. Cuando la amilasa pasa a la sangre se denomina diastasa, también se ubica en órganos importantes como: el páncreas, glándulas salivales y el hígado.

Durante la reacción enzima sustrato se produce una reacción de hidrolisis a nivel de la sangre evitando la formación de polímeros que dificultarían el transporte de otras sustancias y compuesto biomoleculares séricas, la efectividad de los electrolitos depende de la disminución de los polisacáridos, todo esto se puede demostrar in vitro en presencia de un buffer fosfato y un indicador ejecutándose reacciones cualitativas y cuantitativas enzimáticas y colorimétricas.

Mediante la determinación de la amilasa sérica, se puede diagnosticar los niveles altos y bajos de los valores normales, así como las numerosas patologías como la anemia, pancreatitis y peritonitis.

Finalmente mencionar que los objetivos trazados en el presente trabajo de investigación fueron:

Conocer la actividad de la amilasa sanguínea en pacientes aparentemente sanos.

Establecer diferencias comparativas y la significancia correspondiente entre los valores hallados en laboratorio. 
Jesús Javier Ñaccha Urbano

Evaluar y diagnosticar los factores de la actividad enzimática en función a la edad, peso y sexo.

Cuantificar la presencia de amilasa sérica mediante el método de la espectrotometría.

\section{MARCO TEÓRICO}

\section{Antecedentes.}

Las investigaciones sobre la amilasa y el almidón se remontan a las épocas del hombre primitivo, donde se hacia el uso empírico del almidón al consumir sus alimentos que hasta hoy se emplea para fermentar el pan, las bebidas a base de frutas.

Leonardo da Vinci en 1519 comparo la nutrición humana y animal. En 1780 Lavosier reconoce las fermentaciones alcohólicas. Gay Lussac en 1810 deduce la ecuación de la fermentación alcohólica.

Surgiendo la bioquímica con pequeños catalizadores descubiertos 1850 por Peyen y Lersos donde purifican la diastasa o amilasa del trigo, mostrando que era termolábil e hidrolítica. Louis Pasteur en 1864 demuestra que la fermentación era provocada por microorganismo levaduriformes, destruyendo as i la teoría de la generación espontánea. Otswald en 1893 señala que las enzimas son catalizadoras orgánicas y Emil Fisher propone su teoría de la llave y la cerradura que sucede entre una enzima y el sustrato.

Buchner en 1897 descubre que la fermentación alcohólica es producida por la levadura y el azúcar.

En 1913 Michaelis Menten desarrolla la teoría cinética de la acción enzimática.

Summer en 1926 obtuvo la primera enzima denominada ureasa.

El año 1922 Alexander Fleming descubre la lisozima como agente antibacteriano. Desde 1965 la Unión Internacional de Bioquímica (UIB) comienza a investigar y clasificar a base de pruebas enzimáticas utilizando fluidos biológicos, agentes microbianos, inhibidores y marcadores patológicos al final codifico desde el punto de vista molecular. 1966 Monod y Jacob junto a Changeux formulan la teoría de la regulación enzimática.

\section{Características específicas.}

El almidón y el glucógeno son degradada por la hexoquinasa generándose la glucosa 1 fosfato, la cual será convertida en glucosa-6-fosfato por la fosfoglucosa isomerasa. Otra ruta para degradar este sustrato es mediante la alfa amilasa y beta amilasa, obteniéndose un disacárido denominado maltosa y esta su vez se hidroliza hasta la glucosa gracias al maltasa. 
Jesús Javier Ñaccha Urbano

Los ligamentos subterminales de la cadena liberan las dos hexosas terminales, la amilasa es una enzima hidrolítica y digestiva y junto a la lipasa, sacarosa, proteasa y nucleasas. Estructuralmente está compuesto de aminoácidos cuya cantidad y ordenamiento difieren en función a su procedencia.

Asi mismo con tienen por lo menos un átomo gramo de calcio que cumple doble función de mantener la proteasa en una posición especial para dar estabilidad a las estructuras ternarias y cuaternarias.

\section{Actividad fisiológica.}

Sobre el origen de la amilasa pancreática se realiza a nivel de los islotes de Langerhans, a su vez también produce glucagón e insulina, existen pequeños conductos de acinos que transportan hacia los fermentos digestivos, cavidad peritoneal y al entrar en la sangre como dijimos cambia de nombre a diastasa, para luego entrar al hígado y desdoblar el glucógeno. La presencia de glucosa, insulina y glucagón pueden disminuir la amilasa sérica, el cual esta ausente en los recién nacidos, surgiendo casi al tercer mes de vida e incrementándose en la edad adulta.

La amilasa pancreática se desplaza junto a la fracción globulina gamma es absorbida por las venas llegando a los vasos linfáticos y en menor proporción a la cavidad peritoneal.

\section{Actividad enzimática.}

Se refiere a la cantidad de sustrato que cataliza, la cual puede ser efectuado por factores como la concentración de enzimas y sustratos, la temperatura, el $\mathrm{pH}$ y la actividad catalítica.

Para medir la cantidad de una enzima en una muestra biológica o extracto tisular o alguno fluido biológico, se mide la velocidad de reacción catalizada por ella, por el método espectrotométrico en circunstancias apropiadas, según la velocidad y la cantidad tanto de la enzima como del sustrato, pero no es fácil determinar molecularmente la masa y su contenido.

La alfa amilasa es una enzima con un peso molecular de $50 \mathrm{kDa}$ y diferentes autores señalan que las enzimas pertenecen a la familia de las glicohidrolasas, con más diez dominios. El dominio central o catalítico es muy conservado y se conoce como domino $\mathrm{A}$, que consiste en u pliegue simétrico de 8 láminas beta plegadas en forma de barril y rodeadas por 8 hélices alfa o barril tipo TIM). 
Es importante señalar que el el extremo C-terminal de las laminas beta del dominio A se encuentran los residuos aminoácidos conservados que estén implicados en la catálisis y en la unión del sustrato y que este domino se ubica en la región $\mathrm{N}$-terminal de la proteína. MATERIALES Y MÉTODOS MÉTODO

\section{DE ACTIVIDAD DE LA AMILASA DEL GT LAB.}

Fundamento.

La enzima alfa amilasa de la muestra hidroliza el sustrato de almidón. La cantidad de almidón desdoblado para un determinado tiempo, es la medida de la actividad enzimática presente en la muestra. Para la cuantificación fotométrica se utiliza el reactivo yodo, que forma un complejo azul con el almidón, cuya intensidad de color disminuye al aumentar la actividad de amilasa en la muestra.

Amilasa en suero o plasma (colocar nota 2)

\begin{tabular}{|l|l|l|}
\hline & Basal & Muestra \\
\hline Reactivo 1 (Nota 2) & $1 \mathrm{ml}$ & $1 \mathrm{ml}$ \\
\hline \multicolumn{2}{|l|}{ Preincubar unos minutos a3 $7^{\circ} \mathrm{C}$} & $20 \mathrm{ul}$ \\
\hline Muestra & - & $1 \mathrm{ml}$ \\
\hline Mezclar simultáneamente a los 7 minutos \\
\hline Reactivo 2 & $1 \mathrm{ml}$ \\
\hline Mezclar agitando y retirar del baño maría. \\
\hline Agua destilada & $8 \mathrm{ml}$ & $8 \mathrm{ml}$ \\
\hline
\end{tabular}

Cálculos.

Expresados en unidades amilolíticas par suero o plasma

\section{A bs.B-Abs M}

Actividad Amilasa UAldl = x 1000

Abs. B

Valores de Referencia.

Suero o plasma: menor a $120 \mathrm{UA} / \mathrm{dl}$

\section{RESULTADOS}


Tabla 1. Promedio general de la actividad amilásica sérica $U A / d l$ en pacientes altoandinos aparentemente sanos en ambos sexos.

\begin{tabular}{|l|l|l|}
\hline PROMEDIOS & SEXO & SEXO \\
FEMENINO & MASCULINO \\
\hline Amilasa sérica & $125 . \mathrm{UA} / \mathrm{dl}$ & $142 . \mathrm{UA} / \mathrm{dl}$ \\
\hline Edad & 26 años & 28 años \\
\hline Peso & $58 \mathrm{~kg}$ & $64 \mathrm{~kg}$ \\
\hline
\end{tabular}

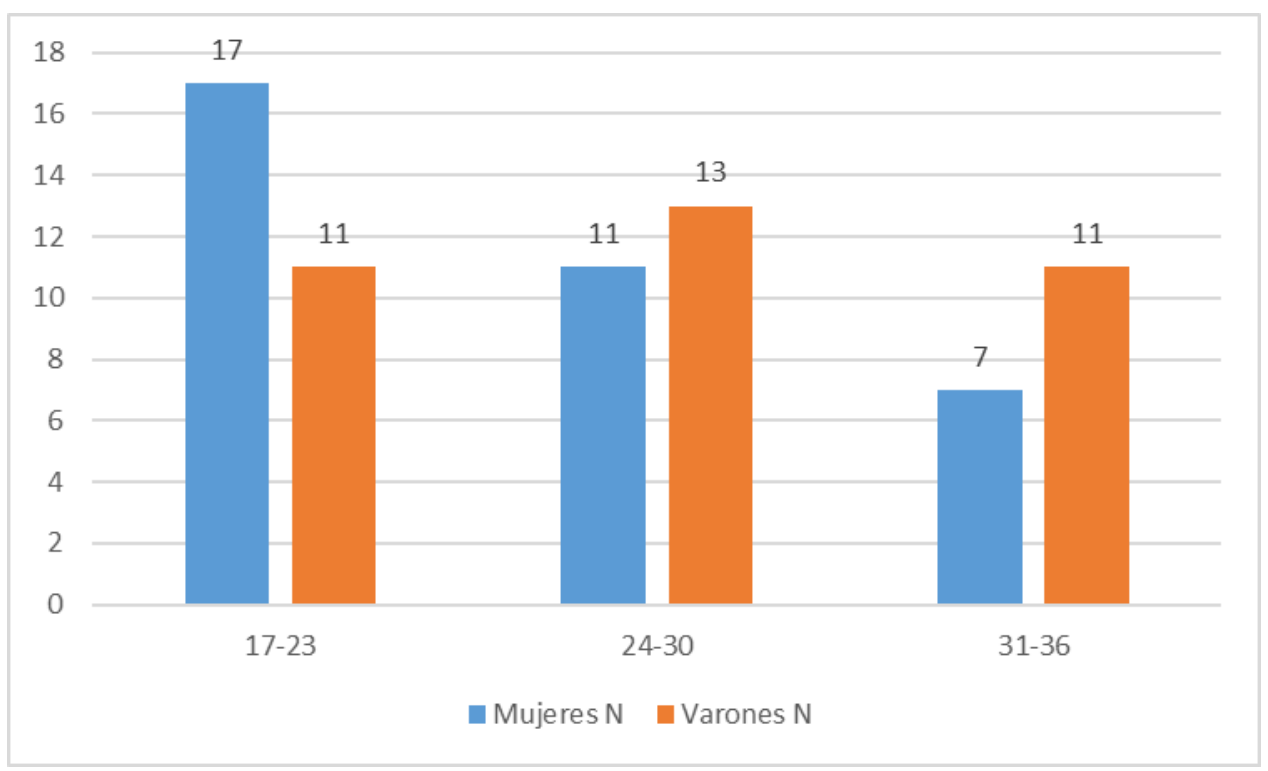

Figura 1. Variación de la actividad amilásica en $\mathrm{UA} / \mathrm{dl}$ pacientes altoandinos aparentemente sanos según los grupos etarios. 


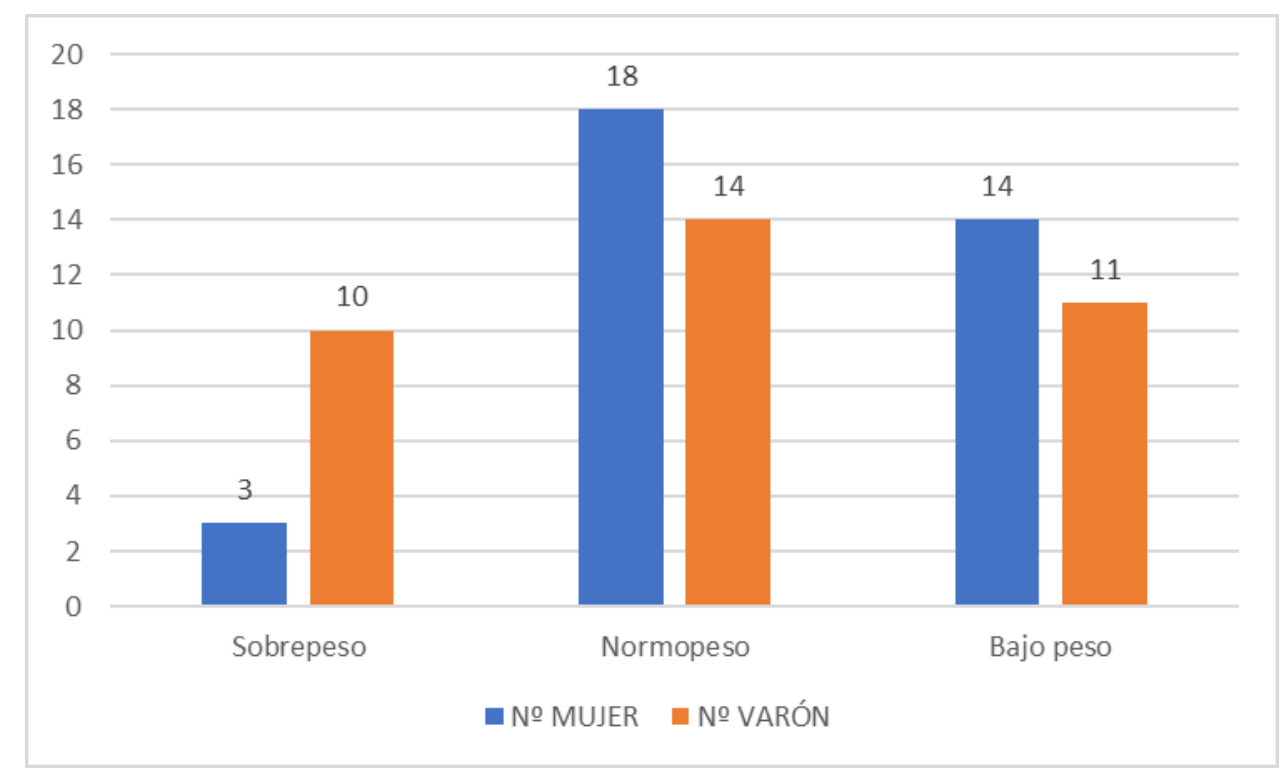

Figura 2. Variación de la actividad amilásica en pacientes altoandinos aparentemente sanos según el índice de masa corporal.

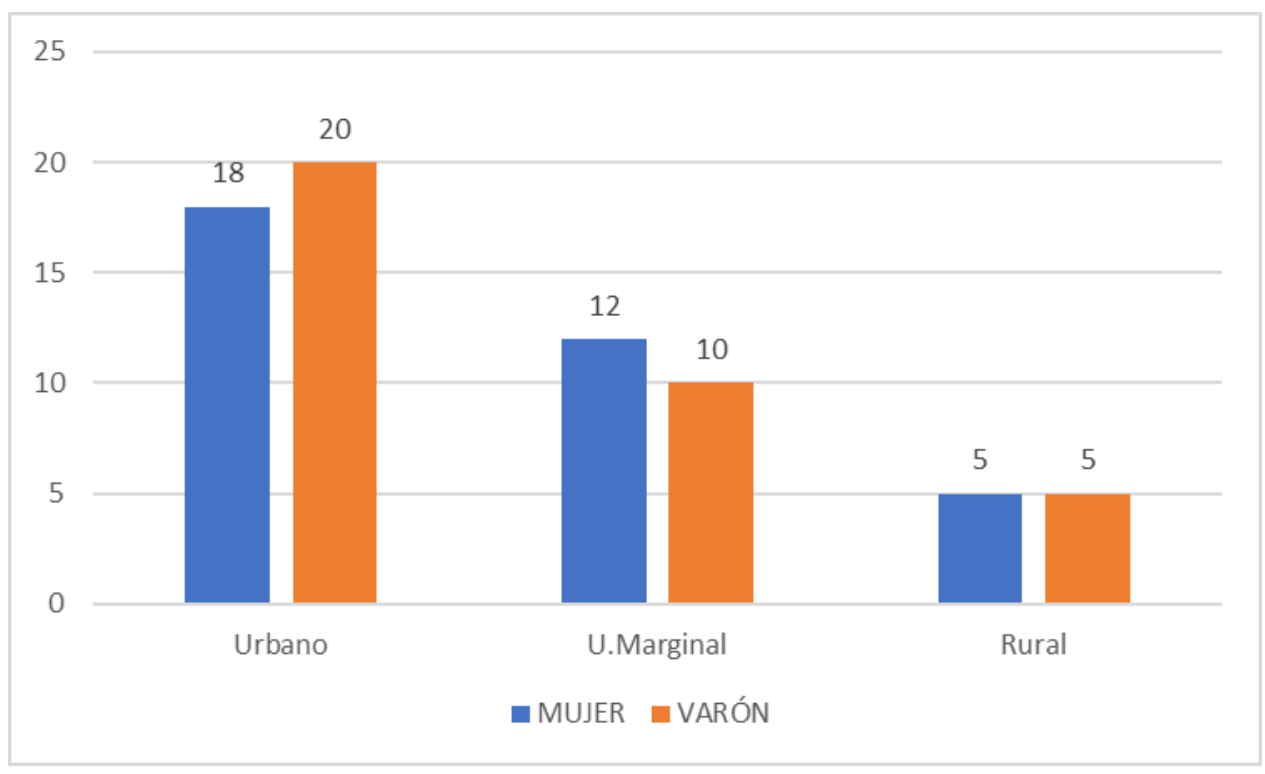

Figura 3. Variación de la actividad amilásica en pacientes altoandinos aparentemente sanos en relación a la procedencia.

\section{DISCUSION:}

Los valores normales de la actividad amilásica sérica es de $120 \mathrm{UA} / \mathrm{dl}$, si comparamos con otros métodos como de Somogy es de 80 a 180 US/ $100 \mathrm{ml}$ y el de Couter de 80 a $150 \mathrm{US} / 100 \mathrm{ml}$. En cuanto a la amilasa urinaria va de 40 a $250 \mathrm{US} /$ hora, normalmente la 
Jesús Javier Ñaccha Urbano

depuración renal equivale a $4 \mathrm{ml}$ de suero/minuto y la medición de la eliminación de amilasa durante una hora con muestras previamente recogidas durante 12 horas.

Es notorio el promedio general obtenido de la actividad amilásica sérica en US/100 ml en pacientes altoandinos aparentemente sanos en la tabla 1. Siendo el sexo femenino con 125 $\mathrm{UA} / \mathrm{dl}$, de 26 años y $58 \mathrm{~kg}$ promedio. Hay un ligero incremento en el peso debido a la ingestión de alimentos a base de carbohidratos especialmente la comida chatarra.

Siendo superior los promedios del sexo masculino con $14 \mathrm{UA} / \mathrm{dl}, 28$ años y $64 \mathrm{~kg}$ de peso, lo que significa que posiblemente se alimentan con carbohidratos presentes en la comida al paso, pero si son bohemios consumidores de las bebidas alcohólicas.

En consideración al grado de amilasa de pacientes altoandinos aparentemente sanos en relación al grupo etario visto en la figura 1, se deduce que la mayor cantidad de mujeres se encuentran en el grupo etario 17 a 23 años con 17 individuos (24.5\%).

En tanto los varones se ubican en gran proporción en el grupo etario de 24 a 30 años con 13 individuos (18.6\%) lo que significa que los varones tienen responsabilidades o familia y trabajan a medio tiempo para cubrir sus necesidades de ahí se interpreta la mayoría de edad que las mujeres.

También encontramos en menor cantidad en el grupo de 31 a 36 años para ambos sexos, que son personas ya tienen una familia o son padres y madres solteras que hacen los esfuerzos posibles por concluir sus estudios.

La determinación de la amilasa sérica en pacientes altoandinos aparentemente sanos en ambos sexos en función al índice de masa corporal se muestra en la figura 2. Podemos observar que personas con sobrepeso son, 3 mujeres (4.3\%) y los 10 varones (14.3\%) lo que indica que esta cantidad es superior a las mujeres consideramos que el sedentarismo y el consumo de comida chatarra y las bebidas alcohólicas lo permiten. Con peso normal o normopeso se encuentra 18 mujeres (25.7\%) y 14 varones (20\%) son jóvenes en actividad física constante. Es preocupante notar personas con bajo peso tal es el caso de 14 mujeres (20\%) y 11 varones (15.7\%) la situación económica que presentan los jóvenes no permite alimentarse bien, cabe mencionar que muchos estudiantes universitarios de bajos recursos por la comida, otros debido a sus quehaceres y deberes se olvidan de alimentarse en su debido momento.

El grado de amilasa en pacientes altoandinos aparentemente sanos en ambos sexos en función a la procedencia se muestra en la figura 3. En su gran mayoría son de procedencia urbano o citadina, 18 mujeres $(25.7 \%)$ y 20 varones (28.6\%) estudian en colegios estatales y particulares de la ciudad, generalmente sus padres son empleados públicos. Ahora 
también hay estudiantes de la zona periférica denominada urbano marginal o barrios populares de la ciudad donde carecen de servicios básicos y son hijos de migrantes, habiendo 12 mujeres (17.2\%) y 10 varones (14.3\%). También encontramos jóvenes provenientes de otras regiones como el VRAEM que están alojados o alquilan algún cuarto, ellos vienen a estudiar para mejorar la calidad de vida de su familia, a veces quedan sin comer por los domingos por no atiende el comedor universitario donde se paga un precio mínimo o no atienden y descansan muchos restaurantes, tratan de agenciarse de alguna fruta o alimento, muchos son jovencitos que recién conocen la ciudad y no tienen familiares, pasan su vida sacrificando su economía.

Podemos precisar la variación de la actividad amilásica en pacientes altoandinos aparentemente sanos de ambos sexos según los grupos etarios en el anexo 4. Notamos que a menos edad 17 a 23 años hay valores de $97.9 \mathrm{UA} / \mathrm{dl}$ para 17 mujeres y $95.6 \% \mathrm{UA} / \mathrm{dl}$ en 11 varones. Incrementándose con la edad de 24 a 30 años en 11 mujeres (98.6 UA/dl), son superados por 13 varones (110.5 UA/dl) pero están dentro de los límites permisibles. El sector de 31 a 36 años de edad ya tiene valores superiores a los normales, por ejemplo, las 7 mujeres presentan (146.4 UA/dl) y 11 varones con $150.6 \mathrm{UA} / \mathrm{dl}$. Estos jóvenes ya casi adultos generalmente son padres jóvenes y tiene que ir a trabajar buscan un tiempo para culminar sus estudios.

A su vez es perceptible la variación de la de la actividad amilásica en pacientes altoandinos aparentemente sanos de ambos sexos, según el índice de masa corporal se plasma en el anexo 5. Los jóvenes son sobrepeso especialmente mujeres presentan valores $162 \mathrm{UA} / \mathrm{dl}$ y los varones $160.4 \mathrm{UA} / \mathrm{dl}$. Estos datos son superiores a los valores normales que va a $120 \mathrm{UA} / \mathrm{dl}$, como mencionamos ellos consumen alimentos a base de carbohidratos y y bebidas alcohólicas, lo que conllevara a la postre en una pancreatitis. Los demás datos se encuentran debajo de los valores normales de las personas con normopeso y bajo peso, mujeres $89.3 \mathrm{UA} / \mathrm{dl}$ y varones con91.9 UA/dl.

\section{CONCLUSIONES}

1. De los 70 pacientes altoandinos, 35 mujeres cuyos promedios son: actividad amilásica fue de $125 \mathrm{UA} / \mathrm{dl}, 26$ años de edad y $58 \mathrm{Kg}$ de peso. Mientras 35 varones con $142 \mathrm{UA} / \mathrm{dl}$ con 28 años y peso de $64 \mathrm{Kg}$, generalmente los datos de los jóvenes del sexo masculino son superiores a los valores normales de $120 \mathrm{UA} / \mathrm{dl}$.

2. En relación al grupo etario se observa en el segmento de 17 a 23 años hay más mujeres 17 (24.3\%) cuyo promedio de actividad amilásica de $97.9 \mathrm{UA} / \mathrm{dl}$. Donde hay más 
Jesús Javier Ñaccha Urbano

varones $13(18.6 \%)$ en el segmento 24 a 30 años con $110.5 \mathrm{UA} / \mathrm{dl}$. El sector de mayor de edad entre 31 a 36 años solamente hay 7 mujeres (11\%) con $146.4 \mathrm{UA} / \mathrm{dl}$ y 11 varones (15.7\%) 150.6 UA/dl, en ambos casos superiores esto debido al consumo de alimentos chatarra y de alcohol por los jóvenes de ambos sexos, son proclives a contraer la pancreatitis. 3. En cuanto a grado de amilasa sanguínea en pacientes altoandinos aparentemente sanos de ambos sexos relación al índice de masa corporal, se muestra que en su mayoría de los jóvenes presentan un normopeso: 18 pacientes $(25.7 \%)$ de mujeres 120.6 UA/dl pero estadísticamente no significativa. Respecto a los 14 varones (20\%) con actividad amilásica $177.7 \mathrm{UA} / \mathrm{dl}$ presenta una pequeña variación de la actividad enzimática, superior a los valores normales.

4. La pancreatitis tiene muchas causas como el consumo excesivo de alcohol, fibrosis quística y los cálculos biliares. los síntomas incluyen náuseas, vómitos, pérdida de peso $\mathrm{y}$ heces grasosas.

\section{BIBLIOGRAFÍA}

Alquel. F. Manual de análisis clínico. Edit. Panamericana S.A. 1977. Buenos Aires.

Bidwell. R. Fisiología vegetal. Edit. AGT. 1978. México.

Bohinsky. B. Bioquímica. Edit. Fondo Educativo Interamericano. 1986. USA.

Carhuaz. R. Capacidad amilásica de dos cepas de Bacillus sp, en diferentes materiales. Tesis UNSCH. 1985. USA.

Guerci. A. Métodos de análisis clínico. Edit. El Ateneo. 1994. Buenos Aires.

Guerrero. E. Determinación de la producción de amilasa en 30 cultivos de Aspergillus niger. VII Congresos Nacional de Biología. 1982. Chiclayo.

Guillen. L. Influencia de 3 medios de cultivo en la producción de el aspegillus sp. XI Congreso Nacional de Biologia. 1994. Tacna.

Guyton. A. Tratado de fisiología médica. Edit. Interamericana S. A. 1985. México.

Haettn. Bioquímica de las levaduras. Edit. Aguilar. 2006. Madrid.

Harper. H. Manual de química fisiológica. Edit. Manual Moderno. 1978. México.

Henley. S. Enzimas en el suero y su valor clínico. Edit. Espaxx. 2004. Buenos Aires.

Iovine. E. Nuevas técnicas en fotocolorimetría clínica. Edit. Universitaria. 1994. Buenos Aires.

Jamardo. N. Química clínica. Edit. Universitaria. 1995. Buenos Aires.

Karrer. P. Traete de chimie organiche. Edit. Ducritton. 1995. París. 
Krup. M. Manual de diagnostico clínico. Edit. Manual moderno. 2000. México.

Lehninger. A. bioquímica. Edit. Omega. 1987. Barcelona.

Lujan. M. Influencia del pH sobre la producción y la actividad de la amiláceas del Bacillus licheniformis. IX Congreso Nacional de Biología. 1994. Tacna.

Lynch. M. Metodos de laboratorio. Edit. Interamericana. 1992. México.

Miranda. H. Producción de amilasa por cinco cultivos de Bacillus sp. VI Congreso Nacional de Biología. 1982. Chiclayo.

Mode. E. Elementos y probabilidades estadísticas. Editorial Reverté. 1970. México.

Orbera. T. Identificación de levaduras de interés biotecnológico. Edit, CEBI. Rev.

Micología. 2004. Santiago de Cuba.

Plummer. D. Bioquímica práctica. Editorial Mc Graw Hill latinoamericana. 1970. Bogotá.

Rovim. E. Química orgánica y bioquímica. Edit. Reverté. 1975. Barcelona.

Stryer. D. Bioquímica. Edit. Reverté 1979. Barcelona.

Tellez. G. Biología aplicada. Editorial Mc Graw Hill latinoamericana. 1991. Bogotá.

Toporek. E. Bioquímica. Editorial Interamericana. 1993. México.

\section{ANEXOS}

Anexo 1. Variación de la amilasa de pacientes altoandinos aparentemente sanos de ambos sexos, según el grupo etario.

\begin{tabular}{|c|c|c|c|c|c|c|}
\hline \multirow[t]{3}{*}{ EDAD } & \multicolumn{4}{|c|}{ NIVELES DE AMILASA } & \multirow{2}{*}{\multicolumn{2}{|c|}{ TOTALES }} \\
\hline & \multicolumn{2}{|c|}{ FEMENINO } & \multicolumn{2}{|c|}{ MASCULINO } & & \\
\hline & $\mathrm{N}^{\mathrm{o}}$ & $\%$ & $\mathrm{~N}^{\mathrm{o}}$ & $\%$ & $\mathrm{~N}^{\mathrm{o}}$ & $\%$ \\
\hline $17-23$ & 17 & 24.3 & 11 & 15.7 & 28 & 40.0 \\
\hline $24-30$ & 11 & 14.7 & 13 & 18.6 & 24 & 34.3 \\
\hline $31-36$ & 7 & 11.0 & 11 & 15.7 & 18 & 25.7 \\
\hline TOTAL & 35 & 50 & 35 & 50 & 70 & 100 \\
\hline
\end{tabular}


Jesús Javier Ñaccha Urbano

Anexo 2. Variación de la actividad amilásica en pacientes altoandinos aparentemente sanos según el índice de masa corporal.

\begin{tabular}{|l|l|l|l|l|l|l|}
\hline \multirow{2}{*}{ PESO } & \multicolumn{2}{l|}{ NIVELES DE AMILASA } & \multicolumn{2}{l|}{ TOTALES } \\
\cline { 2 - 5 } & \multicolumn{2}{|l|}{ FEMENINO } & \multicolumn{2}{l|}{ MASCULINO } & \multicolumn{2}{l|}{} \\
\hline & $\mathrm{N}^{\mathrm{o}}$ & $\%$ & $\mathrm{~N}^{\mathrm{o}}$ & $\%$ & $\mathrm{~N}^{\mathrm{o}}$ & $\%$ \\
\hline Sobrepeso & 3 & 4.3 & 10 & 14.3 & 13 & 18.6 \\
Normopeso & 18 & 25.7 & 14 & 20.0 & 31 & 45.7 \\
Bajo peso & 14 & 20.0 & 11 & 15.7 & 25 & 35.7 \\
\hline TOTAL & 35 & 50 & 35 & 50 & 70 & 100 \\
\hline
\end{tabular}

Anexo 2. Variación de la actividad amilásica en pacientes altoandinos aparentemente sanos según la procedencia.

\begin{tabular}{|c|c|c|c|c|c|c|}
\hline \multirow[t]{3}{*}{ PESO } & \multicolumn{4}{|c|}{ NIVELES DE AMILASA } & \multirow{2}{*}{\multicolumn{2}{|c|}{ TOTALES }} \\
\hline & \multicolumn{2}{|c|}{ FEMENINO } & \multicolumn{2}{|c|}{ MASCULINO } & & \\
\hline & $\mathrm{N}^{\mathrm{o}}$ & $\%$ & $\mathrm{~N}^{\mathrm{o}}$ & $\%$ & $\mathrm{~N}^{\mathrm{o}}$ & $\%$ \\
\hline Urbano & 18 & 25.7 & 20 & 28.6 & 38 & 54.3 \\
\hline Marginal & 12 & 17.2 & 10 & 14.3 & 22 & 31.4 \\
\hline Rural & 5 & 7.1 & 5 & 7.1 & 10 & 14.3 \\
\hline TOTAL & 35 & 50 & 35 & 50 & 70 & 100 \\
\hline
\end{tabular}


Anexo 4. Valores promedios de la actividad amilásica en pacientes altoandinos aparentemente sanos en relación al grupo etario.

\begin{tabular}{|c|c|c|c|c|c|c|}
\hline \multirow{3}{*}{$\begin{array}{l}\text { EDAD } \\
\text { (años) }\end{array}$} & \multicolumn{4}{|c|}{ NIVELES DE AMILASA } & \multirow{2}{*}{\multicolumn{2}{|c|}{ TOTALES }} \\
\hline & \multicolumn{2}{|c|}{ FEMENINO } & \multicolumn{2}{|c|}{ MASCULINO } & & \\
\hline & $\mathrm{N}^{\mathrm{o}}$ & $\ddot{\mathrm{x}}$ & $\mathrm{N}^{\mathrm{o}}$ & $\ddot{\mathrm{x}}$ & $\mathrm{N}^{\mathrm{o}}$ & $\ddot{\mathrm{x}}$ \\
\hline $17-23$ & 17 & 97.9 & 11 & 95.6 & 28 & 96.8 \\
\hline $24-30$ & 11 & 98.6 & 13 & 110.5 & 24 & 104.6 \\
\hline $31-36$ & 7 & 146.4 & 11 & 150.6 & 18 & 148.5 \\
\hline TOTAL & 35 & 144.3 & 35 & 118.9 & 70 & 116.6 \\
\hline
\end{tabular}

Anexo 5. Valores promedios de la actividad amilásica en pacientes altoandinos aparentemente sanos en relación al índice corporal.

\begin{tabular}{|l|l|l|l|l|l|l|}
\hline \multirow{2}{*}{ PESO } & \multicolumn{2}{l}{ NIVELES DE AMILASA } & \multicolumn{2}{l|}{ TOTALES } \\
\cline { 2 - 6 } & \multicolumn{2}{|l|}{ FEMENINO } & \multicolumn{2}{l|}{ MASCULINO } & \multicolumn{2}{l|}{} \\
\hline$(\mathrm{Kg})$ & $\mathrm{N}^{\mathrm{o}}$ & $\ddot{\mathrm{x}}$ & $\mathrm{N}^{\mathrm{o}}$ & $\ddot{\mathrm{x}}$ & $\mathrm{N}^{\mathrm{o}}$ & $\ddot{\mathrm{x}}$ \\
\hline Sobrepeso & 3 & 162.0 & 10 & 160.4 & 13 & 161.2 \\
Normopeso & 18 & 120.6 & 14 & 177.7 & 32 & 119.2 \\
Bajo peso & 14 & 89.3 & 11 & 91.9 & 25 & 90.6 \\
\hline TOTAL & 35 & 124.0 & 35 & 123.3 & 70 & 123.7 \\
\hline
\end{tabular}

